\title{
Detecting responses to treatment with fenofibrate in pedigrees
}

\author{
Svetlana Cherlin ${ }^{1 \dagger}$, Maggie Haitian Wang ${ }^{2 \dagger}$, Heike Bickeböller ${ }^{3 \dagger}$ and Rita M. Cantor ${ }^{{ }^{*}}$ \\ From Genetic Analysis Workshop 20 \\ San Diego, CA, USA. 4-8 March 2017
}

\begin{abstract}
Background: Fenofibrate (Fb) is a known treatment for elevated triglyceride (TG) levels. The Genetics of Lipid Lowering Drugs and Diet Network (GOLDN) study was designed to investigate potential contributors to the effects of Fb on TG levels. Here, we summarize the analyses of 8 papers whose authors had access to the GOLDN data and were grouped together because they pursued investigations into Fb treatment responses as part of GAW20. These papers report explorations of a variety of genetics, epigenetics, and study design questions. Data regarding treatment with $160 \mathrm{mg}$ of micronized Fb per day for 3 weeks included pretreatment and posttreatment TG and methylation levels (ML) at approximately 450,000 epigenetic markers (cytosine-phosphate-guanine [CpG] sites). In addition, approximately 1 million single-nucleotide polymorphisms (SNPs) were genotyped or imputed in each of the study participants, drawn from 188 pedigrees.
\end{abstract}

Results: The analyses of a variety of subsets of the GOLDN data used a number of analytic approaches such as linear mixed models, a kernel score test, penalized regression, and artificial neural networks.

Conclusions: Results indicate that (a) CpG ML are responsive to Fb; (b) CpG ML should be included in models predicting the TG level responses to $\mathrm{Fb}$; (c) common and rare variants are associated with TG responses to $\mathrm{Fb}$; (d) the interactions of common variants and CpG ML should be included in models predicting the TG response; and (e) sample size is a critical factor in the successful construction of predictive models representing the response to Fb.

Keywords: Fenofibrate treatment, GOLDN study, Triglycerides, Epigenetics, Predictive modeling

\section{Background}

The Genetics of Lipid Lowering Drugs and Diet Network (GOLDN) study was designed to investigate potential contributors to the effects of fenofibrate $(\mathrm{Fb})$ on triglyceride (TG) levels [1]. Because of the variety of genetic, genomic, and trait measures, the longitudinal nature of the data encompassing a drug treatment, and the challenges provided by the nonindependence of family members, this rich GAW20 data set led to a broad spectrum of analyses. In addition to the real GOLDN data, simulated data provided the opportunity to formally evaluate

\footnotetext{
* Correspondence: rcantor@mednet.ucla.edu

'Svetlana Cherlin, Maggie Wang and Heike Bickeböller contributed equally to this work.

${ }^{4}$ Department of Human Genetics, David Geffen School of Medicine at UCLA, 695 Charles E. Young Dr. South, Los Angeles, CA 90095, USA

Full list of author information is available at the end of the article
}

statistical approaches. Here we summarize the research questions, study designs, and analytic approaches used by the members of the Genetics of Treatment Response group of 8 papers focused on association and prediction analyses of treatment response. We provide a brief summary of their findings; for a more complete understanding of these investigations, we suggest reading the published manuscripts [2-9].

$\mathrm{Fb}$ is a treatment for elevated TG levels. There are well-known outcomes, although individual responses are highly heterogeneous. Binding of $\mathrm{Fb}$ activates peroxisome proliferator-activated receptor- $\alpha$, starting a cascade that leads to the modulation of genes that regulate lipoprotein metabolism and inflammation. Reduced levels of lipids and lipoproteins, including TGs, are observed after fasting, and even stronger effects are observed after a fatty meal. There are also many nonlipid effects, such

(c) The Author(s). 2018 Open Access This article is distributed under the terms of the Creative Commons Attribution 4.0 International License (http://creativecommons.org/licenses/by/4.0/), which permits unrestricted use, distribution, and reproduction in any medium, provided you give appropriate credit to the original author(s) and the source, provide a link to the Creative Commons license, and indicate if changes were made. The Creative Commons Public Domain Dedication waiver (http://creativecommons.org/publicdomain/zero/1.0/) applies to the data made available in this article, unless otherwise stated. 
as improvements in biomarkers of inflammation. Treatment is often administered to individuals with high lipid levels when changes to diet and exercise either cannot be followed or do not show the desired results [10]. There are many genes involved in the variation in lipids and inflammation, and DNA methylation may play a central role in baseline TG levels, as well as in posttreatment TG levels, by affecting chromatin structure and altering the availability of coding regions in the transcription process [11]. In the GOLDN study, treatment with micronized $\mathrm{Fb}$ consisted of $160 \mathrm{mg}$ per day for 3 weeks.

A number of well-established statistical approaches are available for modeling and/or predicting the treatment response, depending on the goals of the study. These include full longitudinal data analysis, pretreatment and posttreatment analysis, summary statistics, and end-point analyses, including survival analysis. For longitudinal analyses we use full information of the course of the considered outcome, and for the others we can use aggregated or partial information. Often linear mixed models (LMMs) or generalized estimating equations (GEEs) are incorporated to address the correlations within the data. There are several ways regression models can be applied to analyze pretreatment and posttreatment data. Three common models are (a) a follow-up model, (b) a change analysis, and (c) an analysis of covariance (ANCOVA) model. They are:

Follow-up: postTG $=\mathrm{a}+\mathrm{b} \times$ covariates + error Change: postTG - preTG $=\mathrm{a}+\mathrm{b} \times$ covariates + error ANCOVA: postTG $=\mathrm{a}+\mathrm{b} \times$ covariates $+\mathrm{c} \times$ preTG + error

where postTG is posttreatment TG level and preTG is pretreatment TG level. The definition of Change can be based either on a difference or a ratio, depending on whether a log-transformation is applied. A Percent Change model can also be applied, where an appropriate value is between 30 and 50\%, depending on ln preTG [10]. A binary cutoff such as $30 \%$ change, indicating that the responders and nonresponders to treatment can also be applied. An example of a summary analysis is provided by Irvin et al. [12], who used these same GOLDN data to consider the area under the curve of TG at several points in time before and after the high-fat meal.

The focus of most of the 8 manuscripts in this group was predicting the TG level response to $\mathrm{Fb}$ treatment. There were several complexities in these data, including the nonindependence of subjects in the pedigrees and the large number of single-nucleotide polymorphism (SNP) markers, genome-wide. The analytic approaches were fairly broad, and the most common was LMM, which tests for associations between the markers and the trait, while adjusting for the statistical nonindependence of members of the same pedigree. An alternative approach, the kernel score test (KST), used by Yasmeen et al. [9] allows one to test a set of markers for overall association with a trait, using a semiparametric procedure. LASSO (least absolute shrinkage and selection operator) regression used by Cherlin et al. [3] predicts the treatment response from markers based on shrinking regression coefficient toward zero through a penalty on the coefficients. Xia et al. [6] applied an artificial neural network (ANN), a semiparametric modeling technique that does not require linearity.

TG and methylation levels (ML) at approximately 450,000 epigenetic markers were measured pretreatment and posttreatment in the study participants, allowing a wide variety to the analyses. In addition, approximately 1 million SNPs were genotyped or imputed in each of the study participants drawn from 188 pedigrees, permitting the integration of clinical, genetic, and genomic data. Each paper used the SNPs to inform their analyses, however only some used the TG data [3, 4, 6-9] or epigenetic data $[2,5,6,9]$. Most used the full family data, while one paper selected independent individuals from each pedigree [9], and another investigated the impact of approaches to correcting for family structure [4].

Two contributions analyzed the simulated data $[3,9]$. Both knew the answers prior to the analysis and both used the suggested simulated replicate (84). The GAW20 simulated data used the same family structure, genotypes, and pretreatment TG and ML as the real data, with posttreatment TG levels simulated using a model with 5 causal SNPs influencing posttreatment TG levels fully, only when they were unmethylated. These cytosine-phosphate-guanine (CpG) markers, along with another 5 , increased posttreatment TG level variability.

Although SNP and TG level data were analyzed to identify predictors of TG level response by most of the investigators, the inclusion of longitudinal epigenetic and TG level data pretreatment and posttreatment provided an opportunity for interesting research questions, along with some analytic challenges, regarding ML under drug treatment. Research on epigenetic responses to $\mathrm{Fb}$ or other drugs has not been extensive, and the GOLDN study is exceptional in providing these longitudinal treatment response data. ML are highly variable and depend on factors such as age, smoking, treatment, and laboratory/batch/probe effects and cell type used. Here, they were assayed from the CD4+ T cells extracted from whole blood. Because of the widespread effects of lipids and inflammation, it is hypothesized that this cell type should be sufficient in the GOLDN study.

Beta scores, which correspond to the methylated proportion of total signal from the population-specific probe, were provided. Two different probes were used for pretreatment and posttreatment, which had serious implications, producing a confounding batch effect that 
presented analytic challenges for detecting Fb-responsive CpG sites [2] and interpreting methylation quantitative trait loci (meQTL) analyses [5]. To account for the batch effect, Cantor et al. [2] compared the ranks of posttreatment and pretreatment ML familial and variability distributions to detect those $\mathrm{CpG}$ sites exhibiting a likely genetic change in response to treatment with $\mathrm{Fb}$. This was followed by a meQTL analysis of a filtered number of candidate responsive $\mathrm{CpG}$ sites. Wu et al. [5] conducted a full genome cis-meQTL analysis identifying many more such sites, while accounting for 2 different probes.

The most common considerations of all contributions were adjusting for family structure and use of covariates in the analyses. LMM were used by most to adjust for family structure [2, 5-7]. Other approaches included principal components (PCs) [3] or GEE [6], while Yasmeen et al. [9] included only unrelated individuals in the analysis. Hsu et al. [4] investigated different approaches of adjusting for family structure using kinship matrices. In most analyses, the covariates included nongenetic effects such as age, sex, center, smoking status, and a variable number of PCs.

In the subsequent sections, we provide greater detail regarding (a) the primary research questions posed by the 8 studies; (b) the analytic approaches used to investigate them; (c) the data used in the analyses; (d) the primary findings of each study; (e) a discussion of the factors that help us interpret the primary findings; and (f) the conclusions that can be drawn from this collective work. Greater detail on the individual studies can be gleaned from the original manuscripts [2-9].

\section{Methods}

\section{GOLDN study design}

The GOLDN study used a longitudinal design [13, 14] to study the TG level response to Fb. Participants were middle-aged, self-reported white individuals, likely to be genetically homogenous, who were recruited through a previous family study [12] from 2 centers in the United States. Individuals with extreme TG measures and/or a recent history of severe cardiovascular disease were excluded. Participants were asked to fast and abstain from alcohol, and not take lipid-lowering drugs for at least 4 weeks prior to the inception of the study. A high-fat meal challenge was given twice, 3 weeks apart, during which time $\mathrm{Fb}$ treatment was given to lower lipids.

Lipid levels, including TGs, were measured approximately 1 day prior to the drug intervention, with times 1 and 2 corresponding to measurements taken prior to treatment and times 3 and 4 corresponding to measurements taken after the treatment, within each pair (TG1 and TG2, TG3 and TG4) only 1 or 2 days apart. One can assume that TG1 and TG2 as well as TG3 and TG4 measure the same levels, except for random variation. The 8 papers in this group focused on treatment effects by using either T2 or T4, or the means of T1 and T2 or T3 and T4. These are denoted as preTG and postTG, respectively. Because TG levels have a skewed distribution, some authors used log-transformed triglyceride (lnTG).

Genetic markers were array-based SNPs. Epigenetic marker ML were beta-scores of $\mathrm{CpG}$ markers using a methylation array, based on CD4+ T cells and measured only at time points 2 and 4 . The GAW20 simulations used the same data, except that posttreatment TG levels were simulated based on linear models.

\section{Analytic approaches used in the 8 genetics of treatment response contributions}

Our Genetics of Treatment Response groups investigated associations with and predictions of responses to $\mathrm{Fb}$. There is a marked distinction in the aims of association and prediction analyses, although both can begin with the same CpG ML and TG levels data in a single study sample. Association analysis tests each CpG ML to identify those having a significant relationship with TG levels in the population under analysis. The goal is to then use bioinformatics and functional studies to reveal the biology driving these associations. Prediction analysis focuses on using the data in the study sample to develop an analytic model composed of CpG ML in order to predict TG levels. The ultimate goal is to use this prediction model and CPG ML in individuals who have not had their TG levels measured for prediction of their specific TG level. As an example, regression models are widely used for prediction.

Table 1 summarizes the aims of each project and the analytic methods used, in alphabetical order of the first named author. While most contributions concentrated on SNPs and ML as predictors in association analyses [2, 5-7, 9], Yang and Chen [8] used a homozygosity intensity measure. Contributions that investigated the association between the SNPs and the TG treatment response used the full genome-wide data [3, 4, 6-9]. Wu et al. [5] searched for cis-meQTL around each CpG site, genome-wide. Some did more targeted analyses. Cantor et al. [2] tested specific CpG sites showing a likely genetic response to treatment for meQTL. Yasmeen et al. [9] tested TG with genomic regions chosen around causal and noncausal $\mathrm{CpG}$ sites, based on the simulated data. Xia et al. [6] tested a subset of SNPs chosen by GEEs. Most contributions used LMMs to perform association tests [2, 5-7], whereas Yasmeen et al. [9] applied KST and linear regression. Prediction analyses employed penalized regression [3] and ANNs [6] with 10-fold cross validation. Below, we provide details regarding the primary analytic approaches used by the 8 papers in our group. 
Table 1 Primary aims and statistical modeling methods

\begin{tabular}{lll}
\hline First Named Author & Aims of the Analysis & Analytic Methods \\
\hline Cantor & Filter CpG sites for those exhibiting genetic contributions to ML; & $\begin{array}{l}\text { Concordance of familiality and variability of CpG } \\
\text { distributional outliers, LMM }\end{array}$ \\
Cherlin & Predicting TG response to Fb with SNPs & LASSO penalized regression \\
Hsu & Evaluating adjustments for family structure & LMM \\
Wu & Genome-wide cis-meQTL studies & LMM \\
Xia & Evaluate ML in predicting TG response to Fb & ANN, GEE, and LMM \\
Xu & Predicting TG response to Fb with SNPs & LMM and KST \\
Yang & Association between homozygosity intensity and TG response to Fb & GEE \\
Yasmeen & Predicting TG response to Fb with SNPs and CpG ML & KST and linear regression
\end{tabular}

ANN Artificial neural networks, CpG Cytosine-phosphate-guanine, Fb Fenofibrate, GEE Generalized estimating equations, KST Kernel score test, LASSO Least absolute shrinkage and selection operator, $L M M$ Linear mixed models, meQTL Methylation quantitative trait locus, ML Methylation level, SNPs Single nucleotide polymorphisms, TG Triglyceride levels

\section{Linear mixed models}

LMM approaches are widely used in genetic studies of pedigrees. This method assumes that the expected value of a trait is a linear combination of fixed and random effect predictors. Although genetic and covariate effects are modeled as fixed, family effects are considered random effects. LMM can be described as follows:

$$
y=X \beta^{T}+u+\epsilon
$$

where $\boldsymbol{y}$ is a vector of traits; $\boldsymbol{X}$ is the SNP data coded according to the minor allele count; $\boldsymbol{\beta}$ is a vector of the regression coefficients for fixed effects; $\boldsymbol{u}$ is a vector of random effects, $\mathbf{u} \sim N\left(\mathbf{0}, 2 \sigma_{g}^{2} \boldsymbol{\Phi}\right)$, in which $\boldsymbol{\Phi}$ is a matrix of pairwise kinship coefficients; and $\boldsymbol{\epsilon} \sim N\left(\mathbf{0}, \sigma_{\epsilon}^{2} \boldsymbol{I}\right)$ is a vector of the residuals ( $\boldsymbol{I}$ is the identity matrix). The covariance matrix $2 \sigma_{g}^{2} \Phi$ is block-diagonal with 1 block per family. The kinship matrix can be calculated from a known pedigree structure, or from the genetic data when the pedigree structure is not available. Different implementations allow the kinship matrix to be estimated separately from the association testing, thus allowing for the use of alternative packages for kinship matrix estimation when performing association tests. Kinship matrices calculated using different methods tend to differ from each other. There is little difference, however, between the results of the association or prediction analyses obtained using different estimation methods [15].

\section{Kernel score test}

In KST, the trait $\boldsymbol{y}$ is expressed as:

$$
\boldsymbol{y}=\boldsymbol{X} \boldsymbol{\beta}^{T}+h(Z)+\epsilon
$$

where $\boldsymbol{X}$ is a matrix of known fixed covariates; $\boldsymbol{\beta}$ is a vector of the regression coefficients; $\boldsymbol{\epsilon} \sim N\left(\mathbf{0}, \sigma_{\epsilon}^{2} \boldsymbol{I}\right)$ is a vector of residuals; $h(Z)=K \boldsymbol{a}^{T}$ is a nonparametric function that depends on the kernel matrix $K=\boldsymbol{Z} \boldsymbol{Z}^{T}$; and $\boldsymbol{a}$ is a vector of random effects, $\boldsymbol{a} \sim N(\mathbf{0}, \tau \boldsymbol{K})$. The matrix $\boldsymbol{Z}$ is the matrix of the markers, and $\tau$ is the genetic covariance component. KST investigates whether the genetic covariance component equals zero (ie, $\tau=0$ ) [16], which can be interpreted as of test of whether there are aggregated genetic effects contributing to the trait, $y$.

\section{Least absolute shrinkage and selection operator}

LASSO [17] is a penalized regression model.

The trait $y$ is expressed as:

$$
y=X \beta^{T}+\epsilon
$$

where $\boldsymbol{X}$ is a matrix of known fixed covariates; $\boldsymbol{\beta}$ is a vector of the regression coefficients; and $\boldsymbol{\epsilon} \sim N\left(\mathbf{0}, \sigma_{\epsilon}^{2} \boldsymbol{I}\right)$ is a vector of residuals. LASSO allows shrinkage of the estimators of the regression coefficients in a linear model toward zero using a penalty. The estimators of the regression coefficients $\boldsymbol{\beta}$ are found by minimising the sum of the residual sum of squares and a penalty function:

$$
\hat{\beta}_{o}, \hat{\boldsymbol{\beta}}=\operatorname{argmin}\left[\sum_{i=1}^{n}\left(y_{i}-\beta_{o}-\sum_{j=1}^{p} \beta_{j} x_{i j}\right)^{2}+\lambda\|\boldsymbol{\beta}\|_{\ell_{1}}\right]
$$

where $\lambda$ is a regularization parameter that controls the amount of shrinkage, and $\|\beta\|_{\ell_{1}}$ is an $\ell_{1}$-norm penalty which is a sum of the absolute values of the coefficients (ie, $\|\boldsymbol{\beta}\|_{\ell_{1}}=\sum_{j=1}^{p}\left|\beta_{j}\right|[p$ is a number of markers $]$ ). One important property of the LASSO penalty is that it allows the coefficients to be set to exactly zero, thus performing variable selection.

\section{Artificial neural network}

ANN is a computational model based on a collection of nodes that are connected in layers, where the signal travels from the input layer to the output layer, including possible hidden layers. An ANN consists of the interconnections 
between different layers of nodes, the weights of the interconnections, and of the activation function for converting a node's weighed input to its output.

Each layer of an ANN can be described by a neural network function as follows:

$$
f_{i}\left(x_{i}\right)=g\left(\sum_{j} w_{i j} x_{j}+b_{j}\right)
$$

where index $i$ represents the nodes of a layer; index $j$ represents input nodes; $w_{i j}$ and $b_{j}$ are weights; and $g$ is an activation function [18]. Different layers can employ different activation functions. In our group, Xia et al. [6] used a 3-layer ANN with a hyperbolic tangent sigmoid transfer function as an activation function for the hidden layer, and a linear function as an activation function for the output layer. Different algorithms are available for training an ANN. For example, Xia et al. [6] used an adaptive gradient descent with momentum as a training method for ANN.

\section{Study designs used in the genetics of treatment response contributions}

Table 2 summarizes the study designs employed by our 8 GAW20 contributions, in alphabetical order of the first named author. The second column gives the outcomes assessed for the treatment response of that study. For example, in row 1 , Cantor used the top $0.1 \%$ of the ranks of posttreatment ML sibling correlations (sib corrs) and SDs to select the CpG sites likely to be responsive to treatment, followed by a meQTL analysis, while in row 2, Cherlin used the log of the posttreatment TG levels as the predicted treatment response. The third column indicates that both studies used SNPs as predictors, and the fourth column indicates they used pretreatment ML and pretreatment TG levels as baseline measures, respectively. The next three columns indicate whether PCs were used in the analysis, and if so, how many, the covariates used, and how the study addressed family structure. Most contributions adjusted for some covariates as well as between 10 and 20 PCs for SNPs or 4 PCs for ML, and adjusted for kinship via random effects in the model. One contribution investigated sibling pairs to identify likely heritable CpG ML [2], one used only independent individuals [9], and Hsu et al. [4] evaluated the approaches for adjusting for nonindependence of family members, including analyzing independent individuals.

\section{Results}

The investigations we report here are focused on the response to treatment with $\mathrm{Fb}$. However, the study designs and analytic approaches used are quite varied, and the results are fairly broad. Table 3 presents the main results of the studies in alphabetical order of the first named author.

\section{Support for $\mathrm{CpG}$ responses to treatment with $\mathrm{fb}$}

Four studies $[2,5,6,9]$ addressed the role of $\mathrm{CpG}$ sites in response to $\mathrm{Fb}$. Two provided support for a $\mathrm{CpG} \mathrm{ML}$ response to treatment with $\mathrm{Fb}$. A third showed the importance of $\mathrm{CpG} M \mathrm{ML}$ in predicting the TG response to $\mathrm{Fb}$, and a fourth showed that inclusion of SNP-CpG interactions improves the prediction of posttreatment TG levels.

In the first study, Cantor et al. [2] addressed the very fundamental question of whether any $\mathrm{CpG}$ sites are responsive to $\mathrm{Fb}$. Their study design used a novel approach to address the confounding batch effect between pretreatment and posttreatment ML. They searched for those ML reflecting a posttreatment genetic contribution by filtering

Table 2 Design elements of studies addressing fenofibrate treatment effects

\begin{tabular}{|c|c|c|c|c|c|c|}
\hline $\begin{array}{l}\text { First Named } \\
\text { Author }\end{array}$ & Outcome Variable & $\begin{array}{l}\text { Genetic \& Genomic } \\
\text { Predictors }\end{array}$ & Baseline Measures & PCs & Covariates Included & $\begin{array}{l}\text { Treatment of } \\
\text { Family Data }\end{array}$ \\
\hline Cantor & $\begin{array}{l}\text { Post ML sib corrs \& SDs, } \\
\text { meQTL }\end{array}$ & SNPS & Pre ML sib corrs \& SDs & & & LMM \\
\hline Cherlin & $\operatorname{Ln}($ postTG) & SNPS & Ln (preTG) & 20 & Age, center, smoking & PCs \\
\hline Hsu & PreTG & SNPS & & 4 & Age, sex, center & LMM Independents \\
\hline Wu & Ln (postML - preML) & SNPS & & 10 & Age, sex, batch, smoking & LMM \\
\hline \multirow[t]{2}{*}{ Xia } & (PostTG - preTG)/preTG & & PreTG & 10 & Age, sex, smoking, ML & Empirical kinships \\
\hline & Binary TG & & & & & \\
\hline $\mathrm{Xu}$ & PostTG - preTG & SNPS & & 10 & $\begin{array}{l}\text { Age, center, ATP, smoking, } \\
\text { IDF }\end{array}$ & LMM \\
\hline Yang & PostTG - preTG & SNPS & & 10 & $\begin{array}{l}\text { Age, sex, center, ATP, } \\
\text { smoking, IDF }\end{array}$ & GEE \\
\hline Yasmeen & $\operatorname{Ln}($ postTG) & ML, SNPS & Ln (preTG) & none & Age & Independents \\
\hline
\end{tabular}

ATP Adult Treatment Panel, IDF International Diabetes Federation, LMM linear mixed model, $L n$ natural logarithm, meQTL methylation quantitative trait locus, ML Methylation levels, PCs Number of principal components; pre, pretreatment; post, posttreatment, SDs Standard deviations; sib corrs, sibling correlations, SNPs Single-nucleotide polymorphisms, TG Triglyceride levels 
Table 3 Primary results for GAW20 treatment response group

\begin{tabular}{|c|c|}
\hline First Named Author & Results \\
\hline Cantor & $\begin{array}{l}\text { Genetic screening of ML identifies ANAPC2 and KIAA1804 as Fb responsive; rs3087779 and rs1294198 } \\
\text { are meQTL for those genes. }\end{array}$ \\
\hline Cherlin & $\begin{array}{l}\text { LASSO regression on LD-pruned GWAS data provides low prediction power in simulated and real data; } \\
\text { increasing samples to } 7 \mathrm{~K} \text { provides detectable signals and reasonable prediction accuracy. }\end{array}$ \\
\hline Hsu & LMM is the preferable approach when adjusting for family structure. \\
\hline Wu & $\begin{array}{l}\text { Genome-wide studies identify } 229 \text { cis-meQTL for } 610 \text { CpG sites using LMM; rs3733749 and cg00514575, } \\
\text { upstream of MGAT1 is strongest signal. }\end{array}$ \\
\hline Xia & $\begin{array}{l}\text { Adding } \mathrm{CpG} \text { ML to a neural network with SNPs and clinical traits improves prediction of TG response } \\
\text { to Fb by } 4 \% \text {. }\end{array}$ \\
\hline $\mathrm{Xu}$ & TG LMM identifies 4 significant SNPs, including rs964184 previously associated with lipid lowering statins. \\
\hline Yang & MACROD2 homozygosity intensity is associated with the TG response to $\mathrm{Fb}$ using genome-wide GEE. \\
\hline Yasmeen & $\begin{array}{l}\text { Including CpG-SNP interactions improves a KST TG prediction model; previously reported CPT1A is } \\
\text { nominally replicated. }\end{array}$ \\
\hline
\end{tabular}

CpG Cytosine-phosphate-guanine, Fb Fenofibrate, GEE Generalized estimating equation, GWAS Genome-wide association study, KST Kernel score test, LASSO Least absolute shrinkage and selection operator, $L D$ Linkage disequilibrium, $L M M$ Linear mixed models, meQTL Methylation quantitative trait locus, ML Methylation level, SNP Single-nucleotide polymorphism, TG Triglyceride

the posttreatment familiality and variability of ML distributions for outliers. Increased familiality and variability are hallmarks of a genetic effect [19]. Two genes, ANAPC2 and KIAA1804, were selected, and both also had highly significant meQTL, providing support for the existence of Fb-responsive CpG sites. In the second, Wu et al. [5] conducted a very broad genome-wide investigation of cis-meQTL. By using LMM, they identified 229 SNPs associated with ML changes at $610 \mathrm{CpG}$ sites. Among those, there were several consistent with what was reported previously. The most significant, located upstream of MGAT1, is known to be related to TG levels or lipid accumulation [20]. Enrichment analysis using the National Human Genome Research Institute genome-wide association studies (GWAS) catalogue identified 6 SNPs colocalized with 8 previously documented disease loci. Site cg09222892, located in gene RHCE, is associated with a well-known lipid SNP, rs10903129, in the gene TMEM57. These studies provide additional support for the existence of Fb-responsive CpG sites.

In the third study, Xia et al. [6] evaluated the contribution of ML in predicting a 30\% reduction in TG levels using stratified risk modeling and ANN. Including ML in their models reduced the error rate by $4 \%$, indicating that methylation data contributes to prediction accuracy of the drug response. The top predictors, rs10521308 (FTO), rs2206135 (CTNNBL1), cg13438334 (DGAT1), and cg22390041 (ALDH4A1) are located in genes known to be associated with obesity risk. In the fourth study, Yasmeen et al. [9] used simulated posttreatment TG levels to evaluate KST models for identifying associated regions around 5 causal and 5 noncausal CpG sites. Models without SNP-ML interactions were nonsignificant; however, when these interactions were included, significant $p$ values were observed. Their results support the importance of considering the interactions of SNPs and ML when modeling the effects of $\mathrm{Fb}$ on TG levels, and illustrate that KST is appropriate for modeling treatment response with epigenetic data.

\section{Support for common variants in TG-level responses to fb} Two manuscripts provided support for the association of common variants with the TG-level response to $\mathrm{Fb} . \mathrm{Xu}$ et al. [7] identify plausible SNP associations using LMM. Their top SNP, rs964184, is associated with lipid-lowering statin treatment [21]. Gene-based rare variant association testing revealed 6 meeting false discovery rate criteria. In addition, DNMT3L, which is known to regulate DNA methylation activity and is associated with obesity [22], was identified. Yang and Chen [8] conducted a more complex analysis to identify SNPs associated with TG levels. This study investigated homozygosity disequilibrium by identifying nonrandom patterns of homozygosity using homozygous intensity scores, GEE, and a sliding window. This phenomenon has been implicated in both Mendelian and complex diseases. Three regions surrounding rs254239, rs7037978, and rs17704829 provide support for the importance of MACROD2 in the response to $\mathrm{Fb}$.

\section{Analytic and study design considerations in predicting the response to $\mathrm{fb}$}

Two papers focused on analytic questions regarding sample size and correction for the nonindependence of pedigree members. Cherlin et al. [3] explored the predictive ability for drug response by penalized regression methods, providing evidence that a large sample size is needed to achieve good predictions. GWAS using LASSO regression on 680 individuals was conducted on 
posttreatment TG levels in the simulated and real data with pretreatment TG levels as the baseline, resulting in poor prediction. An analysis of a much larger independent data set showed a much better prediction with the same method, suggesting that a sample size of a few thousand individuals is needed to achieve good prediction with LASSO. In the second paper, Hsu et al. [4] evaluated the effect of adjusting for family structure. As expected, only analyzing unrelated subjects, consisting of 1 representative from each family, reduced power substantially, compared to using LMM or treating the pedigree members as independent. These manuscripts provide support for collecting a large sample and using the full sample when analyzing pedigrees.

\section{Discussion}

This manuscript summarizes the aims, methods, study designs and results of 8 GAW20 investigations that were grouped together because they focused on the genetics of responses to treatment with $\mathrm{Fb}$ and the methods to examine it. The questions addressed and methods applied were derived from the longitudinal TG and ML data collected before and after Fb treatment in the GOLDN study. SNP data permitted both targeted and genomewide assessments of genetic associations with TGs. ML data on CpG sites permitted targeted and genome-wide meQTL analyses. Genetics and genomics data on the same individuals undergoing treatment allowed an analysis of their interactions in the prediction of response. Analytic methods, where the number of genetic and genomic predictors is larger than the sample size, were applied, and findings indicate that an adequate sample size is critical. The 8 manuscripts clearly illustrate that correcting for the nonindependence of individuals within pedigrees using LMM to identify SNP associations with $\mathrm{Fb}$ response is straightforward, but correction when developing more complex models is not. This suggests that there is a need for the development of additional methods to accommodate such data.

The variability among individuals in their responses to drug treatment is often ignored, but as medicine moves toward greater precision in caring for patients, this area of investigation will grow. Currently, the complex influences on drug responses are understudied and often unknown, although there are exceptions for those variants exhibiting a Mendelian impact. The work summarized here clearly indicates that the inclusion of genetics and genomics data in a longitudinal drug treatment study is feasible and that such study has the potential to affect the precision of prediction.

The 8 summarized papers explore the genetic and genomic influences on the differences in drug responses among individuals and the appropriateness of study design elements and analytic methods to detect them. For example, the novelty of measuring pretreatment and posttreatment epigenetic ML invited questions regarding the responses of $\mathrm{CpG}$ sites to treatment with $\mathrm{Fb}$, as well as the predictive role of ML changes in the known TG response to $\mathrm{Fb}$. The former studies were hampered by a batch effect between pretreatment and posttreatment ML, and this confounding design element should provide a note of caution to future studies. However, these data and the analyses we report were successful in providing support for the notion that the ML of some $\mathrm{CpG}$ sites respond to $\mathrm{Fb}$. Future studies can be designed to ensure there is no confounding batch effect, and the specific findings identified here can be studied for replication. In addition, given an unforeseen batch effect, a genetic approach to identify candidate CpG sites for meQTL studies, like the one used here [2], may be appropriate.

Study design remains an important issue for drug response work, and a critical issue is the development of adequate samples. As with other studies of complex traits, effect sizes of individual SNPs and CpG sites are likely to be small and difficult to detect. This is especially important when there is genome-wide multiple testing of interactions, and rare variants. In addition to sample size, the nonindependence of pedigree members was a concern. One may posit that the number of samples in the GOLDN pedigrees would provide more statistical power if they were collected on independent individuals. However, family data are likely to be more homogeneous, which can increase statistical power. The studies reported here corrected for the nonindependence of the pedigree members rather than capitalizing on the genetic transmission of information among family members. Although studying the transmission of the drug response in pedigrees is a more attractive approach, having complete data is unlikely because only some pedigree members take the treatment drug.

\section{Conclusions}

Several conclusions are drawable from the 8 GAW20 manuscripts addressing responses to treatment with $\mathrm{Fb}$ summarized here. Regarding genomics, we can conclude that some $\mathrm{CpG} \mathrm{ML}$ are responsive to $\mathrm{Fb}$. In addition, CpG ML should be included in models predicting the TG responses to $\mathrm{Fb}$. Regarding genetic contributions, both common and rare variants are associated with TG responses to $\mathrm{Fb}$. Furthermore, genetics and genomics should be combined to include the interactions of common variants and $\mathrm{CpG}$ ML in models predicting the TG level response to $\mathrm{Fb}$. Regarding study designs, multiple classes of models and statistical analyses are appropriate for these studies, and sample size is a critical factor in the successful construction of predictive models representing the response to $\mathrm{Fb}$. 


\section{Abbreviations}

ANCOVA: Analysis of covariance; ANN: Artificial neural network;

CpG: Cytosine-phosphate-guanine; Fb: Fenofibrate; GAW20: Genetic Analysis Workshop 20; GEEs: Generalized estimating equations; GOLDN: Genetics of Lipid Lowering Drugs and Diet Network; GWAS: Genome-wide association studies; KST: Kernel score test; LASSO: Least absolute shrinkage and selection operator; LMMs: Linear mixed models; meQTL: Methylation quantitative trait locus; ML: Methylation levels; PCs: Principal components; postTG: Posttreatment TG level; preTG: Pretreatment TG level; SNPs: Single-nucleotide polymorphisms; TG: Triglyceride; TG1, TG2, TG3 and TG4: TG measured at times 1,2,3 and 4

\section{Funding}

Publication of the proceedings of Genetic Analysis Workshop 20 was supported by National Institutes of Health grant R01 GM031575. Dr. Cherlin was supported by Wellcome Trust grant 102858/Z/13/Z and MRC/Arthritis Research UK award: Maximizing Therapeutic Utility in RA (MATURA) grant MR-K015346. Dr. Wang was supported by National Science Foundation of China grant 81473035 and CUHK direct grant 4054334. Dr. Bickeböller was supported by DFG German Research Foundation grant KFO 242 BI 576/5-1. Dr. Cantor was supported by the Database and Statistics Core of National Institutes of Health (NIH) grant HL28481.

\section{Availability of data and materials}

The data that support the findings of this study are available from the Genetic Analysis Workshop (GAW) but restrictions apply to the availability of these data, which were used under license for the current study. Qualified researchers may request these data directly from GAW.

\section{About this supplement}

This article has been published as part of BMC Genetics Volume 19 Supplement 1, 2018: Genetic Analysis Workshop 20: envisioning the future of statistical genetics by exploring methods for epigenetic and pharmacogenomic data. The full contents of the supplement are available online at https://bmcgenet.biomedcentral.com/articles/supplements/volume19-supplement-1.

\section{Authors' contributions}

RMC structured and edited the manuscript. SC, MW, HB and RMC developed the tables and wrote the manuscript. All the authors have read and approved the final manuscript.

\section{Ethics approval and consent to participate}

Not applicable.

\section{Consent for publication}

Not applicable.

\section{Competing interests}

The authors declare that they have no competing interests.

\section{Publisher's Note}

Springer Nature remains neutral with regard to jurisdictional claims in published maps and institutional affiliations.

\section{Author details}

'Institute of Genetic Medicine, International Centre for Life, Newcastle University, Central Parkway, Newcastle upon Tyne NE1 3BZ, UK. ${ }^{2}$ Division of Biostatistics, Centre for Clinical Research and Biostatistics, JC School of Public Health and Primary Care, The Chinese University of Hong Kong, Shatin, N.T., Hong Kong SAR, China. ${ }^{3}$ Department of Genetic Epidemiology, University Medical Center, Georg-August University Göttingen, Humboldtallee 32, 37073 Göttingen, Germany. ${ }^{4}$ Department of Human Genetics, David Geffen School of Medicine at UCLA, 695 Charles E. Young Dr. South, Los Angeles, CA 90095, USA.

\section{Published: 17 September 2018}

\section{References}

1. Irvin MR, Zhi D, Aslibekyan S, Claas SA, Absher DM, Ordovas JM, Tiwari HK, Watkins S, Arnett DK. Genomics of post-prandial lipidomic phenotypes in the genetics of lipid lowering drugs and diet network (GOLDN) study. PLoS One. 2014;9(6):e99509.

2. Cantor R, Navarro L, Pan C. Identifying fenofibrate responsive CpG sites. BMC Proc. 2018;12(Suppl 9). https://doi.org/10.1186/s12919-018-0148-3.

3. Cherlin S, Howey RAJ, Cordell HJ. Using penalized regression to predict phenotype from SNP data. BMC Proc. 2018;12(Suppl 9). https://doi.org/10. 1186/s12919-018-0149-2.

4. Hsu Y, Auerbach J, Zheng T, Lo S-h. Coping with family structure in genome-wide association studies: a comparative evaluation. BMC Proc. 2018;12(Suppl 9). https://doi.org/10.1186/s12919-018-0151-8.

5. Wu J, Patel D, Chung J, Zhu C, Lent S, Fisher V, Pitsillides A, Farrer L, Zhang X An efficient analytic approach in genome-wide identification of methylation quantitative trait loci response to fenofibrate treatment. BMC Proc. 2018; 12(Suppl 9). https://doi.org/10.1186/s12919-018-0152-7.

6. Xia X, Weng $H$, Men $\mathrm{R}$, Sun $\mathrm{R}$, Chong $\mathrm{KC}, \mathrm{BCY} Z$, Wang MH. Incorporating methylation genome information improves prediction accuracy for drug treatment responses. BMC Genet. 2018;19(Suppl 1). https://doi.org/10.1186/ s12863-018-0644-5.

7. Xu Z, Duan Q, Cui J, Qiu Y, Jia Q, Wu C, Clarke J. Analysis of genetic and nongenetic factors influencing triglycerides-lowering drug effects based on paired observations. BMC Proc. 2018;12(Suppl 9). https://doi.org/10.1186/ s12919-018-0153-6.

8. $\quad$ Yang $\mathrm{H}$, Chen $\mathrm{C}$. Homozygosity disequilibrium associated with treatment response and its methylation regulation. BMC Proc. 2018;12(Suppl9). https://doi.org/10.1186/s12919-018-0150-9.

9. Yasmeen S, Burger P, Friedrichs S, Papiol S, Bickeboller H. Relating drug response to epigenetic and genetic markers using a region-based kernel score test. BMC Proc. 2018;12(Suppl 9). https:/doi.org/10.1186/s12919-018-0154-5.

10. Rosenson RS. Fenofibrate: treatment of hyperlipidemia and beyond. Expert Rev Cardiovasc Ther. 2008;6(10):1319-30.

11. Irvin MR, Zhi D, Joehanes R, Mendelson M, Aslibekyan S, Claas SA, Thibeault KS, Patel N, Day K, Jones LW, et al. Epigenome-wide association study of fasting blood lipids in the genetics of lipid-lowering drugs and diet network study. Circulation. 2014;130(7):565-72.

12. Irvin MR, Kabagambe EK, Tiwari HK, Parnell LD, Straka RJ, Tsai M, Ordovas JM, Arnett DK. Apolipoprotein E polymorphisms and postprandial triglyceridemia before and after fenofibrate treatment in the genetics of lipid lowering and diet network (GOLDN) study. Circ Cardiovasc Genet. 2010;3(5):462-7.

13. Aslibekyan S, An P, Frazier-Wood AC, Kabagambe EK, Irvin MR, Straka RJ, Tiwari HK, Tsai MY, Hopkins PN, Borecki IB, et al. Preliminary evidence of genetic determinants of adiponectin response to fenofibrate in the genetics of lipid lowering drugs and diet network. Nutr Metab Cardiovasc Dis. 2013; 23(10):987-94.

14. Aslibekyan S, Kabagambe EK, Irvin MR, Straka RJ, Borecki IB, Tiwari HK, Tsai MY, Hopkins PN, Shen J, Lai CQ, et al. A genome-wide association study of inflammatory biomarker changes in response to fenofibrate treatment in the genetics of lipid lowering drug and diet network. Pharmacogenet Genomics. 2012;22(3):191-7.

15. Eu-Ahsunthornwattana J, Miller EN, Fakiola M, Jeronimo SM, Blackwell JM, Cordell HJ. Comparison of methods to account for relatedness in genome-wide association studies with family-based data. PLoS Genet. 2014;10(7):e1004445.

16. Malzahn D, Friedrichs S, Rosenberger A, Bickeboller H. Kernel score statistic for dependent data. BMC Proc. 2014;8(Suppl 1):S41.

17. Tibshirani R. Regression shrinkage and selection via the LASSO. J R Stat Soc Series B Stat Methodol. 1996;58(1):267-88.

18. Bridges M, Heron EA, O'Dushlaine C, Segurado R, Morris D, Corvin A, Gill M, Pinto C. Genetic classification of populations using supervised learning. PLoS One. 2011;6(5):e14802.

19. Falconer DS, Mackay TFC. Introduction to Quantitative Genetics, 4th ed. Harlow. Harlow: Longman; 1996.

20. Lee YJ, Ko EH, Kim JE, Kim E, Lee H, Choi H, Yu JH, Kim HJ, Seong JK, Kim KS, et al. Nuclear receptor PPARgamma-regulated monoacylglycerol Oacyltransferase 1 (MGAT1) expression is responsible for the lipid accumulation in diet-induced hepatic steatosis. Proc Natl Acad Sci U S A. 2012;109(34):13656-61.

21. Barber MJ, Mangravite LM, Hyde CL, Chasman DI, Smith JD, McCarty CA, Li X, Wilke RA, Rieder MJ, Williams PT, et al. Genome-wide association of lipid-lowering response to statins in combined study populations. PLoS One. 2010;5(3):e9763.

22. Kamei Y, Suganami T, Ehara T, Kanai S, Hayashi K, Yamamoto Y, Miura S, Ezaki O, Okano M, Ogawa Y. Increased expression of DNA methyltransferase $3 \mathrm{a}$ in obese adipose tissue: studies with transgenic mice. Obesity (Silver Spring). 2010;18(2):314-21. 